\title{
Eine Top-Uni für eine Top-Karriere: Wer profitiert?
}

\section{Thomas Schiller}

\section{Relevanz}

Das Renommee der Eliteuniversitäten ist enorm. Ihre Alumni schaffen es in die Top $1 \%$ der Einkommen und dominieren das Who is Who in Wirtschaft und Gesellschaft. Doch wer profitiert von der Eliteausbildung? Ist Talent entscheidend, oder die Abstammung von einem einflussreichen Elternhaus? Fördern die Eliteuniversitäten die Chancengleichheit und den sozialen Aufstieg, oder tragen sie gar zur Zementierung der Ungleichheit bei? Es scheint, dass die Bedeutung von Beziehungen für die Karriere nicht in allen Studiengängen gleich wichtig ist. Der Erfolg einer Management-Karriere hängt scheinbar mehr von Beziehungen ab als in anderen Studienrichtungen. Die Pflege des Beziehungsnetzes profitiert von einem vorteilhaften sozialen Hintergrund und geht von ganz alleine. Umso grösser ist die Herausforderung der Politik, diesen Vorteil zu kompensieren und auf Chancengleichheit hinzuwirken, und der Eliteuniversitäten, ihr Beziehungsnetzwerk allen zu öffnen.

\section{Quelle}

Zimmerman, Setz D. (2019), Elite Colleges and Upward Mobility to Top Jobs and Top Incomes, American Economic Review 109, 1-47.

T. Schiller $(\bowtie)$

Universität St.Gallen, St.Gallen, Schweiz

E-Mail: thomas.schiller@student.unisg.ch 
Eine gute Ausbildung verspricht sozialen Aufstieg. Ein Studienabschluss von einer renommierten Universität steigert die Chance auf einen attraktiven Arbeitsplatz sowie ein hohes Einkommen, nicht selten am obersten Ende der Einkommensverteilung. Viele Universitäten werben mit der Karrierelaufbahn ihrer Absolventen und mit den beruflichen Erfolgen ihrer Alumni um die neue Studierendentalente.

Gibt es tatsächlich einen kausalen Zusammenhang zwischen der Alma Mater und der späteren Position ihrer Alumni in der Einkommensverteilung? Wer profitiert am meisten von einem Studium an einer Eliteuniversität? Kann ein Studium Chancengleichheit und sozialen Aufstieg fördern oder zementiert es bloß die bestehende Ungleichheit? Wie unterscheiden sich die Studiengänge einer renommierten Universität in ihren Auswirkungen auf Karrieren und Einkommen? Verspricht ein Wirtschaftsstudium mehr als eine andere Studienwahl?

Seth Zimmerman von der Universität Chicago untersuchte, wie sich die Ausbildung an einer Eliteuniversität auf Berufs- und Einkommenschancen auswirkt. Dazu verwendete er Daten aus Chile, welche für diese Frage besonders geeignet sind. In Chile müssen alle Bewerber an Spitzenuniversitäten einen standardisierten Aufnahmetest ablegen, der über die Zulassung entscheidet. Damit wird es möglich, Einkommen und Karrieren jener Bewerber, welche die Aufnahme gerade noch schafften, mit dem beruflichen Erfolg der anderen $\mathrm{zu}$ vergleichen, die knapp nicht zugelassen wurden und auf durchschnittliche Universitäten ausweichen mussten. Da beide Bewerbergruppen nahe an der Zulassungsgrenze landeten, ist es sehr plausibel, dass sich ihre Merkmale nicht systematisch voneinander unterscheiden, außer eben durch die Zulassung. Dieser Vergleich ermöglichte es dem Forscher, die Effekte des Studiums an einer Spitzenuniversität unbeeinflusst von anderen Faktoren wie z. B. den intellektuellen Fähigkeiten des Bewerbers zu quantifizieren.

In Chile verfügen die Absolventen eines rechts- und ingenieurwissenschaftlichen sowie eines wirtschaftswissenschaftlichen Studiums an einer Eliteuniversität über die besten Karriere- und Einkommenschancen. Dies trifft im Besonderen auf ein Wirtschaftsstudium zu, das auf eine Tätigkeit im Management vorbereitet: Zwar bewerben sich nur rund $1,8 \%$ aller Studieninteressierten für einen solchen Studiengang, jedoch machen diese rund $27 \%$ aller Top $1 \%$ - und gar $39 \%$ aller Top 0,1\%-Einkommen aus. Die Wahrscheinlichkeit, ein Top 0,1\%-Einkommen zu erreichen und somit mehr zu verdienen als $99,9 \%$ der Bevölkerung, ist mit einem auf Management orientierten Studiengang rund 16mal höher als beispielsweise bei einem Medizinstudium. Ähnliches zeigt sich bei der Berufslaufbahn: Absolventen eines Wirtschaftsstudiums sind überproportional in Führungspositionen vertreten. 
Die empirischen Schätzungen zeigen, dass die Zulassung zu einem Management-Studium an einer Eliteuniversität die Chancen auf ein Höchsteinkommen an der Spitze der Einkommensverteilung und auf eine Führungsposition deutlich verbessert. Ein Bewerber, der knapp in einen solchen Studiengang aufgenommen wurde, hat eine deutlich bessere Chance, zukünftig ein Top 0,1\%-Einkommen zu erzielen, als einer, der knapp abgelehnt wurde und auf die nächst bessere Alternative ausweichen musste. Die Eliteausbildung erhöht die Wahrscheinlichkeit eines Höchsteinkommens um rund $50 \%$ im Vergleich zur Ausbildung an einer durchschnittlichen Universität. Ebenso nimmt dadurch die Wahrscheinlichkeit, im Laufe der Karriere eine Führungsposition zu erreichen, um rund $44 \%$ zu.

\footnotetext{
Absolventen einer Spitzenuniversität haben eine um 50 Prozent höhere Chance, ein Top $0.1 \%$-Einkommen zu erzielen, und eine um 44 Prozent höhere Chance auf eine Führungsposition.
}

Allerdings sind die Vorteile einer Eliteausbildung sehr einseitig verteilt: Überwiegend profitieren männliche Bewerber, die vor dem Studium ein privates Gymnasium besuchten und typischerweise aus wohlhabenden Familien stammen. In dieser Gruppe steigert ein Wirtschaftsstudium an einer Spitzenuniversität die Chance auf ein Top-0,1\% Einkommen um ganze $69 \%$ und auf eine Führungsposition um $54 \%$. Sowohl für Frauen als auch für Männer mit einem öffentlichen Gymnasialabschluss findet der Forscher hingegen keine signifikanten Effekte. Abb. 1 illustriert die Wahrscheinlichkeit eines Spitzeneinkommens abhängig vom
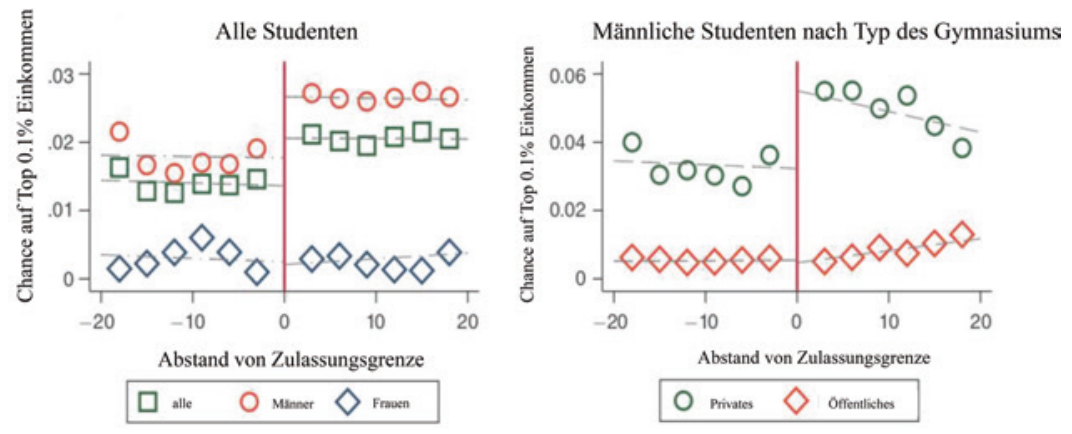

Abb. 1 Wahrscheinlichkeit eines Spitzeneinkommens (Top 0,1\%) und Zulassung zur Eliteuniversität. (Quelle: Zimmerman 2019, S. 24) 
Ergebnis des Aufnahmetests abhängig vom Geschlecht (links) bzw. Schulbildung (rechts, nur männliche Bewerber). Nur Bewerber mit Testergebnissen über der Schwelle von null wurden zum Studium zugelassen.

Eine Spitzenausbildung begünstigt vorwiegend Männer aus wohlhabenden Familien. In dieser Gruppe steigert das Studium die Wahrscheinlichkeit für ein Höchsteinkommen um 69 Prozent.

Warum profitieren überwiegend Männer aus wohlhabenden Familien vom Studium an einer Eliteuniversität? Seth Zimmerman betrachtet zuerst einige naheliegende Faktoren, welche diese Entwicklung erklären könnten, wie Unterschiede in der Ausbildungsqualität zwischen privaten und öffentlichen Schulen oder Charakteristika des Arbeitsmarktes, welche bestimmte Gruppen begünstigen. Jedoch haben die Absolventen öffentlicher und privater Gymnasien in Chile nahezu identische Testergebnisse in Kernfächern wie Lesen und Mathematik. Solche Faktoren können also die überproportionalen Einkommens- und Karrierevorteile von Männern mit hohem sozioökonomischem Status kaum erklären.

Dass nur bestimmte Gruppen mit hohem Status von der Spitzenausbildung profitieren, scheint eine Besonderheit von Managementkarrieren $\mathrm{zu}$ sein. Seth Zimmerman analysiert als Vergleich auch die Einkommenschancen von Bewerbern medizinischer Studiengänge an Eliteuniversitäten, die ähnlich selektiv sind. Dabei zeigt sich, dass die Zulassung zum Studium die Chance, zu den Top $10 \%$ oder Top 0,1\% der Einkommen aufzusteigen, für Absolventen von Privatschulen nicht signifikant stärker erhöht als für Absolventen öffentlicher Schulen. Die Vorteile einer Spitzenausbildung sind gleichmäßiger verteilt.

Weshalb verlaufen Karrieren im Management anders? Ein zentraler Unterschied zu anderen Tätigkeiten besteht in der Rolle persönlicher Beziehungen, welche den Karriereverlauf oft entscheidend fördern. Solche Bindungen entstehen oft bereits zwischen Kommilitonen an der Universität. Offenbar können in Chile Männer mit einer Privatschulbildung leichter solche Bindungen zu Kommilitonen mit ähnlichem Hintergrund entwickeln. Seth Zimmerman analysiert, wie häufig Absolventen desselben Jahrgangs und derselben Universität gleichzeitig Führungspositionen im selben Unternehmen innehaben. Die Wahrscheinlichkeit dafür ist mehr als doppelt so hoch, wenn jemand zuvor ein privates Gymnasium besucht hat.

Männer mit hohem sozioökonomischem Status formen persönliche Bindungen an der Universität leichter und profitieren daher überproportional von einer Spitzenausbildung in Wirtschaft und Management. 
Ein Studium an einer Eliteuniversität verbessert die Chancen auf Höchsteinkommen und auf Führungspositionen besonders im Management. Davon profitieren allerdings überwiegend Absolventen mit hohem sozioökonomischem Status wie z. B. Männer aus wohlhabenden Familien. Frauen oder Männern aus einfacheren Verhältnissen bringt hingegen eine Eliteausbildung kaum signifikant bessere Karriere- und Einkommenschancen, im Vergleich zu einer ähnlichen Ausbildung an anderen Universitäten. Die Studienergebnisse deuten darauf hin, dass die an der Universität aufgebauten Beziehungen ein wesentlicher Grund für einen besseren Karriereerfolg sind. Männern aus reichem Hause können solche Beziehungen leichter entwickeln und profitieren daher überproportional stark von der Eliteausbildung. Mögliche Lösungsansätze, um die Gewinne gleichmäßiger $\mathrm{zu}$ verteilen und die Chancengleichheit zu verbessern, liegen in einer stärkeren sozialen Integration aller Studierenden während der Ausbildung, beispielsweise durch die zufällige Zuteilung von Mitbewohnern oder Lerngruppen.

Open Access Dieses Kapitel wird unter der Creative Commons Namensnennung 4.0 International Lizenz (http://creativecommons.org/licenses/by/4.0/deed.de) veröffentlicht, welche die Nutzung, Vervielfältigung, Bearbeitung, Verbreitung und Wiedergabe in jeglichem Medium und Format erlaubt, sofern Sie den/die ursprünglichen Autor(en) und die Quelle ordnungsgemäß nennen, einen Link zur Creative Commons Lizenz beifügen und angeben, ob Änderungen vorgenommen wurden.

Die in diesem Kapitel enthaltenen Bilder und sonstiges Drittmaterial unterliegen ebenfalls der genannten Creative Commons Lizenz, sofern sich aus der Abbildungslegende nichts anderes ergibt. Sofern das betreffende Material nicht unter der genannten Creative Commons Lizenz steht und die betreffende Handlung nicht nach gesetzlichen Vorschriften erlaubt ist, ist für die oben aufgeführten Weiterverwendungen des Materials die Einwilligung des jeweiligen Rechteinhabers einzuholen.

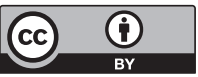

